\title{
Low-pressure phase diagram of crystalline benzene from quantum Monte Carlo
}

Sam Azadi, and R. E. Cohen

Citation: The Journal of Chemical Physics 145, 064501 (2016); doi: 10.1063/1.4960434

View online: https://doi.org/10.1063/1.4960434

View Table of Contents: http://aip.scitation.org/toc/jcp/145/6

Published by the American Institute of Physics

\section{Articles you may be interested in}

Chemical accuracy from quantum Monte Carlo for the benzene dimer

The Journal of Chemical Physics 143, 104301 (2015); 10.1063/1.4930137

High-pressure hydrogen sulfide by diffusion quantum Monte Carlo

The Journal of Chemical Physics 146, 084503 (2017); 10.1063/1.4976836

A climbing image nudged elastic band method for finding saddle points and minimum energy paths

The Journal of Chemical Physics 113, 9901 (2000); 10.1063/1.1329672

Phase Diagram of Benzene to $35 \mathrm{kbar}$

The Journal of Chemical Physics 55, 793 (1971); 10.1063/1.1676145

High pressure solid phases of benzene. I. Raman and x-ray studies of $\mathrm{C}_{6} \mathrm{H}_{6}$ at $294 \mathrm{~K}$ up to $25 \mathrm{GPa}$

The Journal of Chemical Physics 89, 4255 (1988); 10.1063/1.454809

Crystal structure prediction using ab initio evolutionary techniques: Principles and applications

The Journal of Chemical Physics 124, 244704 (2006); 10.1063/1.2210932

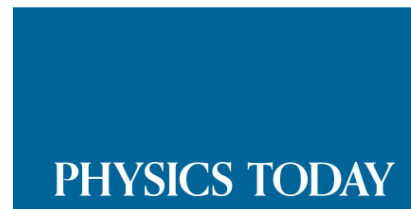




\title{
Low-pressure phase diagram of crystalline benzene from quantum Monte Carlo
}

\author{
Sam Azadi ${ }^{1, a)}$ and R. E. Cohen ${ }^{2}$ \\ ${ }^{1}$ Departments of Physics and Astronomy, University College London, Thomas Young Center, \\ London Centre for Nanotechnology, London WC1E 6BT, United Kingdom \\ ${ }^{2}$ Extreme Materials Initiative, Geophysical Laboratory, Carnegie Institution for Science, Washington, \\ DC 20015, USA; Department of Earth-and Environmental Sciences, Ludwig Maximilians Universität, \\ Munich 80333, Germany; and Department of Physics and Astronomy, University College London, \\ London WC1E 6BT, United Kingdom
}

(Received 29 March 2016; accepted 20 July 2016; published online 8 August 2016)

\begin{abstract}
We studied the low-pressure ( $0-10 \mathrm{GPa}$ ) phase diagram of crystalline benzene using quantum Monte Carlo and density functional theory (DFT) methods. We performed diffusion quantum Monte Carlo (DMC) calculations to obtain accurate static phase diagrams as benchmarks for modern van der Waals density functionals. Using density functional perturbation theory, we computed the phonon contributions to the free energies. Our DFT enthalpy-pressure phase diagrams indicate that the Pbca and $P 2_{1} / c$ structures are the most stable phases within the studied pressure range. The DMC Gibbs free-energy calculations predict that the room temperature $P b c a$ to $P 2_{1} / c$ phase transition occurs at 2.1(1) GPa. This prediction is consistent with available experimental results at room temperature. Our DMC calculations give $50.6 \pm 0.5 \mathrm{~kJ} / \mathrm{mol}$ for crystalline benzene lattice energy. Published by AIP Publishing. [http://dx.doi.org/10.1063/1.4960434]
\end{abstract}

\section{INTRODUCTION}

Molecular crystals, including organic and inorganic, are vital in understanding the physics and chemistry of the Earth and planets. They are also of considerable technological interest. Low-Z molecular systems are among the most abundant in the solar system, as represented by planetary gases and ices. Their behaviour at high pressures is crucial in modelling the structure, dynamic, and evolution of the large planets. Moreover, compression of molecular systems provides the opportunities to form new materials, possibly with novel properties, such as high-temperature superconductivity and disordered and amorphous materials. One of the simplest organic molecular solids is crystalline benzene with aromatic van der Waals (vdW) interactions. Given its simplicity, high symmetric, and rigid molecular structure, crystalline benzene has become the model structure for calculating the lattice model vibrations in molecular crystals. Benzene has been extensively studied theoretically and experimentally. ${ }^{1-4}$ However, the phase transitions and intermolecular interactions are still controversial. The main goal of this paper is to present a comprehensive study of the phase transition of crystalline benzene at low pressures.

Early experiments by Bridgman ${ }^{5}$ revealed that liquid benzene crystallises at $68 \mathrm{MPa}$ with $\mathrm{Pbca}$ space group symmetry and closest C-C intermolecular distance of $3.5 \AA$. This structure, also, was confirmed at zero pressure and $270 \mathrm{~K}^{6,7}$ This phase I is also stable at lower temperatures of 218 and $138 \mathrm{~K}^{8}$ Since then, two experimental phase diagrams have been proposed for crystalline benzene. First,

\footnotetext{
a)s.azadi@ucl.ac.uk
}

based on the phase diagram suggested by Thiéry and Léger, ${ }^{1}$ liquid benzene crystallises at room temperature and pressure 700 bar within an orthorhombic structure $\mathrm{Pbca}$, which is labeled as phase I. Phase II was suggested to exist between 1.4 and $4 \mathrm{GPa}$. Phases I and II primitive unit cells contain four benzene molecules $(Z=4)$. Phase III is stable between 4 and $11 \mathrm{GPa}$. The symmetry of phase III is $P 2_{1} / c$ with two benzene molecules per monoclinic primitive unit cell $(Z=2)$. Second, the phase diagram developed by Ciabini et al. ${ }^{9,10}$ which based on it phase $\mathrm{I}$ is orthorhombic $\mathrm{Pbca} \mathrm{Z}=4$ and phase II is monoclinic $P 2_{1} / c \mathrm{Z}=2$. $^{3}$ Their results are obtained by means of infrared spectroscopy and $\mathrm{X}$-ray analysis under high pressure. The $P 2_{1} / c$ phase is stable up to pressures 20-25 GPa. This phase diagram only consists of two phases (I and II), and this same result has been reported by other experiments. ${ }^{11}$ Katrusiak et al. ${ }^{11}$ have determined the crystal structures of phases I and II at $295 \mathrm{~K}$. The results of their study confirm the phase diagram of Ciabini et al. and show that the structures of phases I and II are Pbca Z $=4$ and $P 2_{1} / c \mathrm{Z}=2$, respectively. The results also indicate the absence of other benzene phases in the pressure range up to $5 \mathrm{GPa}$.

The crystalline benzene phase diagram is a challenge for first-principles theory because the energy differences are insignificant, and they are governed by vdW interactions. The energy difference between crystalline benzene and its lowenergy polymorphs under pressure is less than few $\mathrm{kJ} / \mathrm{mol}$. Metadynamics calculations predict seven phases ${ }^{4}$ as phases I (Pbca Z = 4), I' (Cmca Z = 4), II $\left(P 4_{3} 2_{1} 2 \mathrm{Z}=4\right)$, III $\left(P 2_{1} / c \mathrm{Z}=2\right)$, III' $(C 2 / c \mathrm{Z}=4)$, IV (Pbam Z $\left.=4\right)$, and $\mathrm{V}\left(P 2_{1} \mathrm{Z}=2\right)$. In their calculations, they have used numerous randomly generated metastable crystal structures as starting points for the metadynamics. A few metadynamics 
steps are often sufficient to obtain a more stable structure, which most of the time is similar to $P b c a \mathrm{Z}=4$ or $P 2_{1} / c$ $\mathrm{Z}=2$. Density functional theory (DFT) has also been used to compute the lattice energy of crystalline benzene. ${ }^{12,13}$ Wen et al. employed DFT formalism and used Perdew-BurkeErnzerhof (PBE) ${ }^{14}$ exchange correlation functionals to study the phase diagram of crystalline benzene up to $300 \mathrm{GPa}^{2}$ They explained the complexities observed in benzene at high pressure. In the moderate pressure regime $(\mathrm{P}<20 \mathrm{GPa})$, they found that the $P b c a$ structure is stable up to $4 \mathrm{GPa}$, the $P 4_{3} 2{ }_{1} 2$ phase is preferred in the pressure range of 4-7 GPa, and the $P 2_{1} / c$ structure shows the lowest enthalpy at higher pressures. Therefore, they labelled the Pbca, $P 4_{3} 2_{1} 2$, and $P 2_{1} / c$ structures as phases I, II, and III, respectively. The present study shows that the $P 4_{3} 2_{1} 2$ structure is unstable in the pressure range of $0-10 \mathrm{GPa}$. Thus, the $P b c a$ and $P 2_{1} / c$ structures are labelled as phases I and II, respectively.

Recently, quantum chemistry methods have been applied to benzene to obtain sub-kilojoule/mole accuracy in the lattice energy for crystalline benzene. ${ }^{15}$ Tremendous measures are necessary to obtain such accuracy. In this work, we will show that quantum Monte Carlo (QMC) is an alternative efficient approach to achieve or surpass such accuracy in benzene crystals, as we previously demonstrated for the benzene dimer. ${ }^{16}$

Quantum Monte Carlo (QMC), which approximately solves the electronic Schrödinger equation stochastically, ${ }^{17}$ can yield highly accurate energies for atoms, ${ }^{18,19}$ molecules, ${ }^{20-22}$ and crystals. ${ }^{23-25}$ Previous studies have shown that diffusion quantum Monte Carlo (DMC) can provide accurate energies for noncovalent interactions systems. ${ }^{26-30} \mathrm{DMC}$ can also produce an accurate description of the phase diagram of materials under pressure. ${ }^{31-33}$ In general, QMC-based methods are faster than the most accurate post-Hartree-Fock schemes for large number of particles $\mathrm{N}$. The computational cost of QMC methods scales usually as $\mathrm{N}^{3}-\mathrm{N}^{4}$ depending on the method.

We have demonstrated that QMC can provide chemical accuracy for the benzene dimer system. ${ }^{16}$ We have found optimal variational quantum Monte Carlo (VMC) and DMC binding energies of $-2.3(4)$ and $-2.7(3) \mathrm{kcal} / \mathrm{mol}$. The best estimate of the $\operatorname{CCSD}(\mathrm{T}) / \mathrm{CBS}$ limit is $-2.65(2) \mathrm{kcal} / \mathrm{mol}{ }^{34}$ The consistency among our results, experiments, and quantum chemistry methods is an important sign of the capability of the QMC-based methods to provide an accurate description of weak intermolecular interactions based on vdW dispersive forces.

In this study, we examine the $Z=4$ to $Z=2$ phase transition of crystalline benzene at low pressures. We consider the $P b c a$ and $P 4_{3} 2_{1} 2$ structures as best candidates for $\mathrm{Z}=4$ and the $P 2_{1} / c$ structure for $\mathrm{Z}=2$. We study pressures below $10 \mathrm{GPa}$. We obtain static and dynamic phase diagrams where the phonon contribution to the free energy is included. We employ different vdW functionals ${ }^{35}$ and compare them with conventional DFT functionals. We perform QMC calculations to obtain the static enthalpy-pressure phase diagram of crystalline benzene. We will show that DMC provides accurate results for the phase diagram of crystalline benzene.

\section{COMPUTATIONAL DETAILS}

Given that the energy differences between crystalline benzene structures are small, the calculations must be performed with the highest possible numerical precision. Our DFT calculations were carried out within the pseudopotential and plane-wave approach using the Quantum ESPRESSO suite of programs. ${ }^{36}$ All DFT calculations used ultrasoft pseudopotentials. ${ }^{37}$ Pseudopotentials were obtained by $\mathrm{PBE}^{14}$ exchange correlation functionals. We used a basis set of plane waves with an energy cutoff $100 \mathrm{Ry}$. Geometry and cell optimisations employed a dense $12 \times 12 \times 12$ k-point mesh. The quasi-Newton algorithm was used for cell and geometry optimisation, with convergence thresholds on the total energy and forces of $0.01 \mathrm{mRy}$ and $0.1 \mathrm{mRy} / \mathrm{Bohr}$, respectively, to guarantee convergence of the total energy to less than $1 \mathrm{meV} /$ proton and the pressure to less than $0.1 \mathrm{GPa} /$ proton.

To include the effects of zero point energy (ZPE), vibrational frequencies were calculated using densityfunctional perturbation theory as implemented in Quantum ESPRESSO ${ }^{36}$ The ZPE per proton at a specific cell volume $V$ was estimated within the quasi-harmonic approximation: $E_{\mathrm{ZPE}}(V)=\hbar \bar{\omega} / 2, \quad$ where $\bar{\omega}=\sum_{\mathbf{q}} \sum_{i=1}^{N_{\text {mode }}} \omega_{i}(\mathbf{q}) /\left(N_{\mathbf{q}} N_{\text {mode }}\right)$. $N_{\text {mode }}$ and $N_{\mathbf{q}}$ are the numbers of vibrational modes in the simulation cell and phonon wave vectors $\mathbf{q}$, respectively, and the summation over $\mathbf{q}$ includes all k-points on a $2 \times 2 \times 2$ grid in the Brillouin zone.

The thermodynamic properties are determined by the Helmholtz free energy $F=E-T S$. The free energy can be written as the sum of an electronic and a vibrational term. The electronic entropy is negligible for insulators: $F_{e l} \simeq E_{e l}$. In our calculations, the electronic part $E_{e l}$ is obtained using the DMC method. Thus, the main quantity to calculate for obtaining the thermal properties and finite temperature phase diagram is the vibrational free energy $F_{p h}$. We use quasi-harmonic approximation to calculate the vibrational free energy, ${ }^{38}$

$$
F_{p h}(T, V)=k_{B} T \sum_{i, \mathbf{q}} \ln \left\{2 \sinh \left[\hbar \omega_{i, \mathbf{q}}(V) / 2 k_{B} T\right]\right\},
$$

where $k_{B}, V$, and $\omega_{i, \mathbf{q}}$ are Boltzmann constant, unit cell volume, and eigenvalue of the phonon Hamiltonian, respectively. The pressures $P$ are calculated from the Helmholtz free energies by $P=-(\partial F / \partial V)_{T}$.

We used the casino code ${ }^{39}$ to perform fixed-node DMC simulations with a trial wave function of the Slater-Jastrow (SJ) form,

$$
\Psi_{\mathrm{SJ}}(\mathbf{R})=\exp [J(\mathbf{R})] \operatorname{det}\left[\psi_{n}\left(\mathbf{r}_{i}^{\uparrow}\right)\right] \operatorname{det}\left[\psi_{n}\left(\mathbf{r}_{j}^{\downarrow}\right)\right],
$$

where $\mathbf{R}$ is a $3 N$-dimensional vector of the positions of the $N$ electrons, $\mathbf{r}_{i}^{\uparrow}$ is the position of the $i$ th spin-up electron, $\mathbf{r}_{j}^{\downarrow}$ is the position of the $j$ th spin-down electron, $\exp [J(\mathbf{R})]$ is a Jastrow factor, and $\operatorname{det}\left[\psi_{n}\left(\mathbf{r}_{i}^{\uparrow}\right)\right]$ and $\operatorname{det}\left[\psi_{n}\left(\mathbf{r}_{j}^{\downarrow}\right)\right]$ are Slater determinants of spin-up and spin-down oneelectron orbitals. These orbitals were obtained from PBE-DFT calculations performed with the plane-wave-based Quantum ESPRESSO code, ${ }^{36}$ employing Trail-Needs ${ }^{40,41}$ Hartree-Fock pseudopotentials. We evaluated the total energy in DMC using the localization approximation. ${ }^{42}$ For the QMC study of $\mathrm{C}$ and $\mathrm{CH}$-based systems, the Hartree-Fock description of the core 
is more accurate. ${ }^{43}$ A detailed study of silicon also showed ${ }^{44}$ that Hartree-Fock provides the most accurate description of the core density compared with generalised gradient approximation and local density approximation (LDA).

We selected a very large basis-set energy cutoff of 200 Ry to approach the complete basis-set limit. ${ }^{45}$ The plane-wave orbitals were transformed into a localised "blip" polynomial basis. ${ }^{46}$ Our conventional Jastrow factor consists of polynomial one-body electron-nucleus, two-body electronelectron, and three-body electron-electron-nucleus terms, the parameters of which were optimised by minimising the variance of the local energy at the VMC level. ${ }^{47,48}$ Our DMC calculations were performed at two different time steps 0.01 and 0.02 a.u. The target population control is two times larger for time step 0.02 a.u. We extrapolated our DMC energies to zero time step using a linear fitting.

\section{RESULTS AND DISCUSSION}

\section{A. Geometry analysis}

In this section, we discuss the results of our geometry optimization. We study the evolution of benzene molecule distances by increasing the pressure. The structure optimization results are compared with experiments.

The primitive unit cells of the $P b c a, P 4_{3} 2_{1} 2$, and $P 2_{1} / c$ structures of solid benzene contain four, four, and two benzene molecules, respectively, as shown in Figure 1. The Pbca and $P 4_{3} 2_{1} 2$ structures have orthorhombic and tetragonal primitive unit cells, respectively, whereas the $P 2_{1} / c$ primitive unit cell is monoclinic.

For geometry analysis of $\mathrm{Z}=4$ and $\mathrm{Z}=2$ structures, we focus on the Pbca and $P 2_{1} / c$. We will show in Sec. III B that these two structures are the best candidates for the phases I $(Z=4)$ and II $(Z=2)$. Our structure optimization indicates that the molecular orientations do not change significantly within the studied pressure range. We calculated the distances between $\mathrm{C}$ atoms on nearest-neighbour (nn) benzene molecules. The $\mathrm{nn} \mathrm{C}-\mathrm{C}$ distances between molecules as function of pressure are reported in Figure 2. The nn C-C distances for $P b c a$ and $P 2_{1} / c$ structures are calculated using $\mathrm{vdW}^{50,52}$ and conventional DFT functionals.

The vdW functionals, particularly vdW-DF2 ${ }^{50} \mathrm{nn} \mathrm{C}-\mathrm{C}$ distances, are in good agreement with experiment. ${ }^{9,10}$ The differences between vdW-DF $1^{52}$ and vdW-DF2 $2^{50} \mathrm{nn} \mathrm{C-C}$ distances reduce with increasing pressure. The PBE nn $\mathrm{C}-\mathrm{C}$ distances are close to $\mathrm{vdW}$ functional results at lower pressures, whereas the differences between PBE and vdW results increase with increasing pressure. The PBE nn C-C distances at higher pressures are close to LDA results. The BLYP nn $\mathrm{C}-\mathrm{C}$ distances are the largest at low pressures. However, BLYP nn C-C distances are more similar to vdW results at pressures larger than $5 \mathrm{GPa}$.

The van der Waals radius of carbon atom $r_{W C}$ is $1.7 \AA$. In crystalline benzene, the benzene molecules are held together by van der Waals forces. The nearest that two $C$ atoms belonging to different benzene molecules can approach each other is estimated by the sum of $r_{W C}$. We calculated the difference between nearest-neighbour (nn) $\mathrm{C}-\mathrm{C}$ distances and the sum of $r_{W C}\left(\delta_{\mathrm{C}-\mathrm{C}}\right)$. Figure 3 illustrates $\delta_{\mathrm{C}-\mathrm{C}}$ for $\mathrm{Pbca}$ and $P 2_{1} / c$ structures. The results are obtained by vdW and conventional functionals. At the same pressure, all the functionals give larger $\delta_{\mathrm{C}-\mathrm{C}}$ for $P 2_{1} / c$ structure. Our equation of state (EOS) calculations, which are presented in Figure 7, indicate that at the same pressure molecular density of $P 2_{1} / c$ is larger than Pbca. LDA and BLYP provide the smallest and largest $\delta_{\mathrm{C}-\mathrm{C}}$. Consequently they yield the smallest and largest $\mathrm{vdW}$ radii for $\mathrm{C}$ atom. Unlike the other functionals, the BLYP $\delta_{\mathrm{C}-\mathrm{C}}$ decline rapidly with increasing the pressure. According to LDA, $P b c a$ results, benzene molecules are strongly bonded at pressures larger than $0.2 \mathrm{GPa}$. In lower pressures, PBE $\delta_{\mathrm{C}-\mathrm{C}}$ is close to $\delta_{\mathrm{C}-\mathrm{C}}$ obtained by $\mathrm{vdW}$ functionals. With increasing the pressure, PBE results become closer to LDA. According to vdW-DF1 results, the benzene molecules in $\mathrm{Pbca}$ structure are bonded above $3 \mathrm{GPa}$, whereas vdW-DF2 results indicate that bonding between benzene molecules in $\mathrm{Pbca}$ phase could happen around $2 \mathrm{GPa}$. Based on the experimental phase diagram, ${ }^{9,10}$ the $P b c a$ phase is stable at pressures below $1.4 \mathrm{GPa}$. Our vdW $\delta_{\mathrm{C}-\mathrm{C}}$ results show that there are no strong bonds between benzene molecules in Pbca phase. In the Pbca

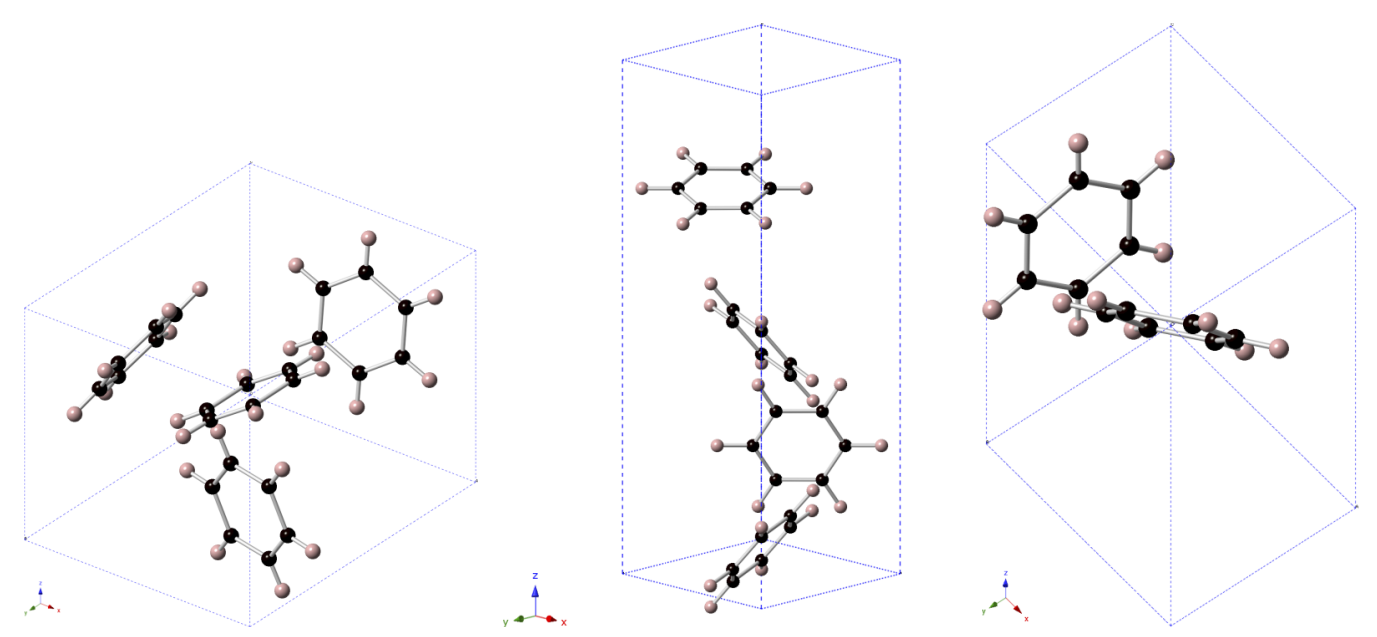

FIG. 1. Unit cells of the $P b c a$ (left), $P 4_{3} 2_{1} 2$ (middle), and $P 2_{1} / c$ (right) structures of solid benzene at low-pressure range. The $P b c a$ and $P 4_{3} 2_{1} 2$ primitive unit cells contain four benzene molecules $(Z=4)$, whereas the $P 2_{1} / c$ structure has two benzene molecules $(Z=2)$ in a monoclinic primitive unit cell. 

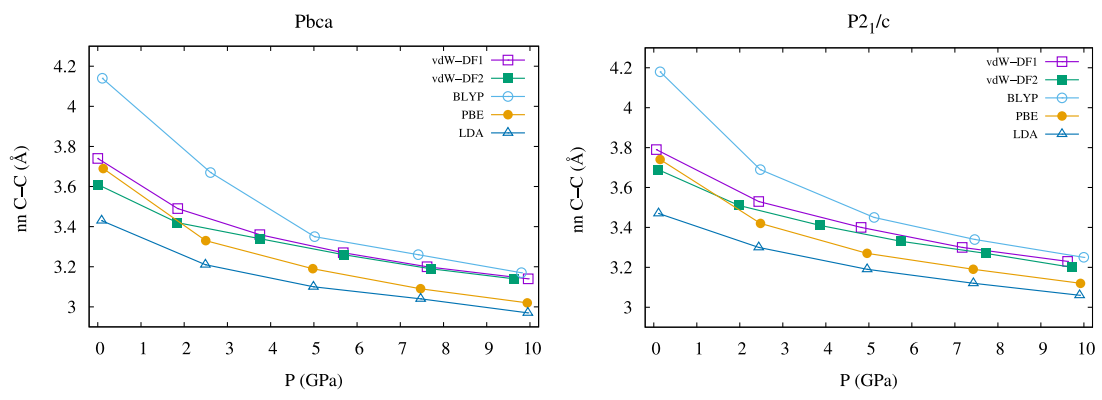

FIG. 2. Pressure evolution of nearestneighbour (nn) $\mathrm{C}-\mathrm{C}$ distances for $\mathrm{Pbca}$ and $P 2_{1} / c$. The results are obtained by $\mathrm{vdW}$ and conventional functionals.
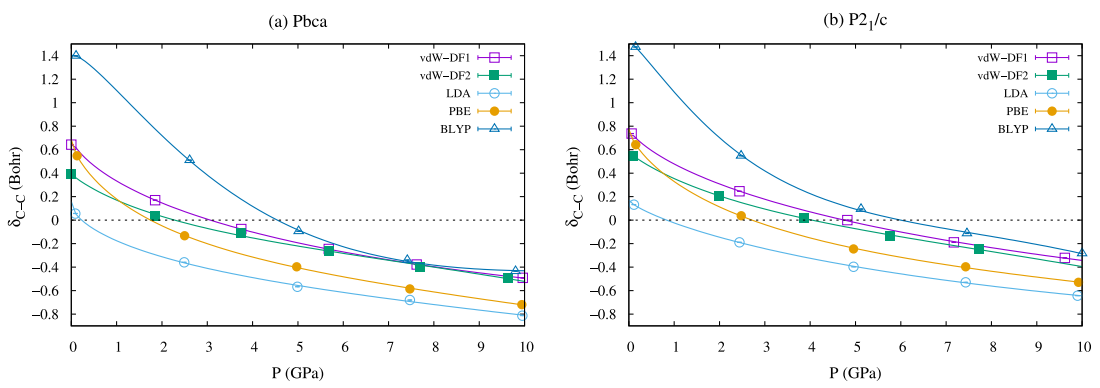

FIG. 3. Pressure evolution of the difference between nearest-neighbour (nn) $\mathrm{C}-\mathrm{C}$ distances and the sum of van der Waals radii of $\mathrm{C}$ atoms $\left(\delta_{\mathrm{C}-\mathrm{C}}\right)$. The results are obtained for $P b c a$ and $P 2_{1} / c$ structures using vdW and conventional functionals.

structure, the benzene molecules only interact through weak dispersive forces.

\section{B. Ground state DFT phase diagram}

We begin our phase diagram study by DFT enthalpypressure calculations at zero temperature. We first present our static phase diagram results where the Born-Oppenheimer (BO) approximation is used. According to BO approximation, the electronic and nuclear wave functions can be separated. At the static level, it is also assumed that the nuclei are infinitely massive and the total nuclear momentum contribution in the Hamiltonian is zero. To find out the best candidate for $\mathrm{Z}=4$ at the studied pressures, we used the $\mathrm{PBE}^{49}$ and vdW$\mathrm{DF} 2^{50}$ functionals to calculate the enthalpy difference between the $P b c a, P 4_{3} 2_{1} 2$, and $P 2_{1} / c$ structures. We performed calculations at six different volumes corresponding to DFT pressures of $0,2,4,6,8$, and $10 \mathrm{GPa}$ (Figure 7). Based on the linear fitting of the PBE results on two enthalpy-pressure points at $\mathrm{P}=0$ and $10 \mathrm{GPa}$, the $P b c a$ structure is stable up to $3.6 \mathrm{GPa}$, whereas $P 4_{3} 2_{1} 2$ is stable in the pressure range of 3.6-6.8 GPa, and finally, the $P 2_{1} / c$ structure has lowest enthalpy in pressures higher than $6.8 \mathrm{GPa}$.

A line between these two enthalpy-pressure points gives excellent agreement with the previous PBE computations by Wen et $a .^{2}$ (Fig. 2(a)). However we find this result to be inaccurate, and a denser set of points in this pressure range is needed.

Using the Vinet ${ }^{51}$ equation of state (EOS), we found that the $P 4_{3} 2_{1} 2$ structure is not stable in the pressure range of $0-10 \mathrm{GPa}$. The results of our EOS calculations are presented in Figure 7. The enthalpy difference between the Pbca, $P 4_{3} 2_{1} 2$, and $P 2_{1} / c$ structures versus pressure is calculated using PBE and vdW-DF2 $2^{50}$ functionals (Figure 4(b)). The root-mean-square deviations (RMSDs) for PBE $-P 2_{1} / c$ and PBE $-P 4_{3} 22_{1} 2$ fittings are 0.0021 and 0.0019 , respectively. The RMSDs for vdW-DF2 $-P 2_{1} / c$ and vdW-DF2 $-P 4_{3} 2_{1} 2$ fittings are 0.0024 and 0.0026 , respectively. We find that instability of $P 4_{3} 2_{1} 2$ is independent of employed functional. Our results indicate that $P b c a$ and $P 2_{1} / c$ are the most stable structures in the studied pressure ranges. These results are consistent with the experimental phase diagram proposed by
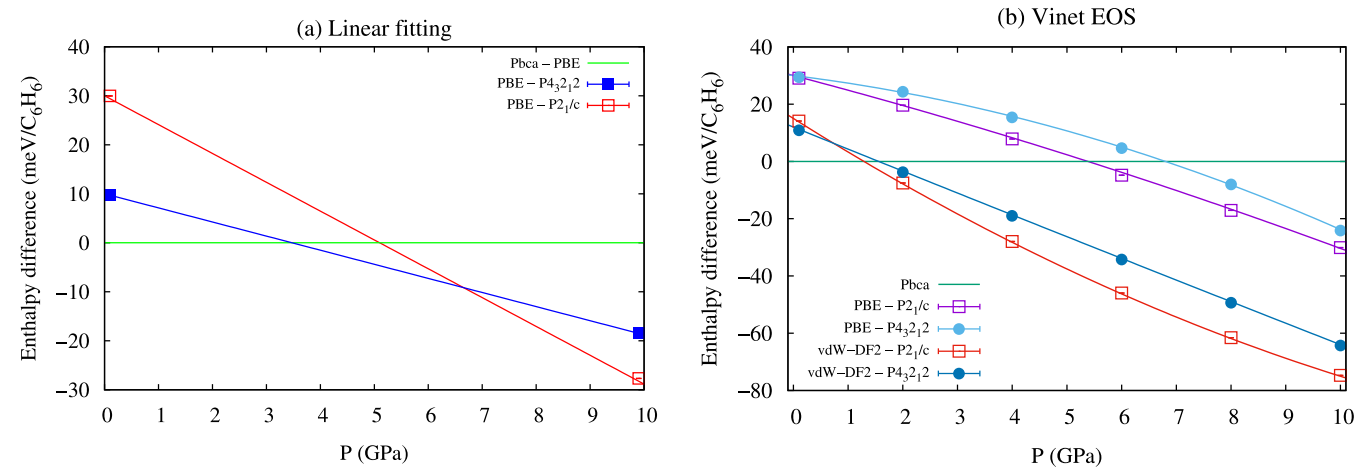

FIG. 4. Enthalpy difference between the $P b c a, P 4_{3} 2_{1} 2$, and $P 2_{1} / c$ structures as function of applied pressure. (a) The results are calculated using PBE and linear fitting on two enthalpy-pressure points at $\mathrm{P}=0$ and $10 \mathrm{GPa}$. (b) The phase diagram is simulated using DFT-PBE and vdW density functional of vdW-DF2. We used the Vinet EOS and six enthalpy-pressure points at $\mathrm{P}=0,2,4,6,8$, and $10 \mathrm{GPa}$. 

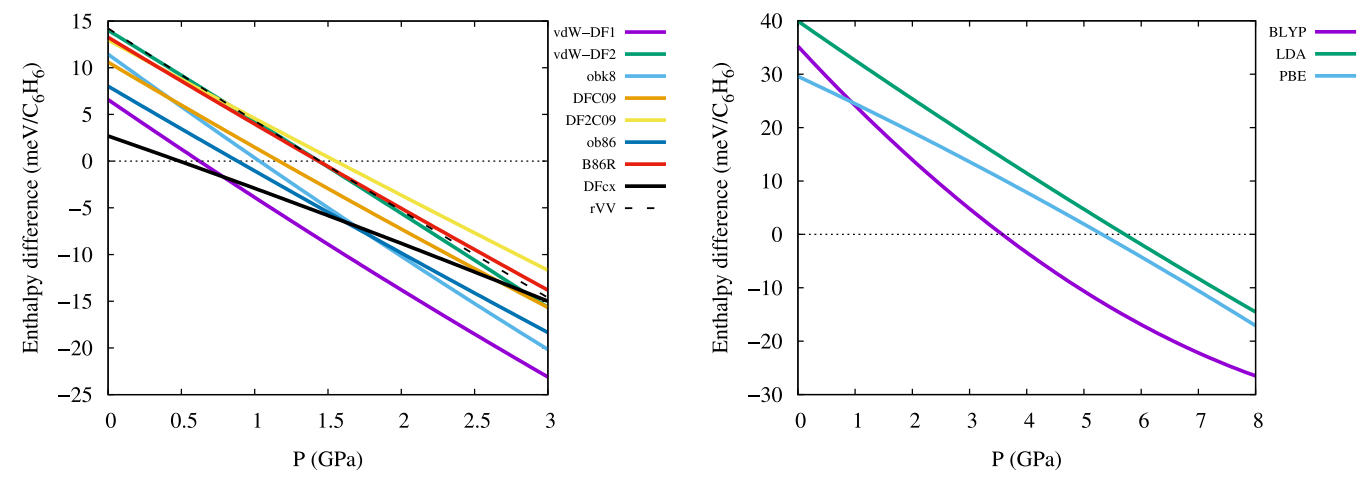

FIG. 5. Enthalpy difference between $P b c a$ (phase I) and $P 2_{1} / c$ (phase II) as function of pressure obtained with vdW and conventional DFT functionals. The left panel shows the results of vdW-DF1, ${ }^{52} \mathrm{vdW}-\mathrm{DF} 2,{ }^{50} \mathrm{rVV},{ }^{56,57}$ obk8, B86R, ob86, $,{ }^{35,53} \mathrm{DFCx},{ }^{55} \mathrm{DFC} 09$, and DF2C09 ${ }^{54} \mathrm{vdW}$ functionals. The right panel illustrates the results of conventional DFT including PBE, ${ }^{49} \mathrm{LDA},{ }^{58}$ and BLYP. ${ }^{59}$

Ciabini et al. ${ }^{3,9-11}$ Therefore, in the rest of this paper, we label $P b c a$ and $P 2_{1} / c$ as phases I and II, respectively.

To study the importance of dispersion effects, we calculated the phase diagram of crystalline benzene using different functionals (Figure 5). We employed vdW-DF1, ${ }^{52} \mathrm{vdW}-\mathrm{DF} 2,{ }^{50}$ vdW-DF-obk8, vdW-DF-ob86, vdW-DF2-B86R, ${ }^{35,53}$ vdWDF-C09, vdW-DF2-C09, ${ }^{54}$ vdW-DF-cx,${ }^{55}$ and vdW-rVV ${ }^{56,57}$ $\mathrm{vdW}$ functionals. Except $\mathrm{rVV}$ functional, the nonlocal term in the other vdW functionals is either vdW-DF1 ${ }^{52}$ or vdW-DF2 ${ }^{50}$ Employing various gradient corrections to the exchange energy results in a variety of vdW functionals. We also determined the phase diagram using conventional DFT functionals, including PBE ${ }^{49} \mathrm{LDA},{ }^{58}$ and BLYP. ${ }^{59}$

The vdW functionals yield different I-II phase transition pressure. Figure 6 illustrates $P b c a$ to $P 2_{1} / c$ phase transition pressures which are obtained by different vdW functionals. The $\mathrm{Cx}^{55}$ and DF2C0954 functionals show the lowest and highest phase transition pressures, respectively. The difference between largest and smallest phase transition pressure is about $1.1 \mathrm{GPa}$. This value corresponds to inaccuracy in prediction of phase transition pressure by vdW functionals. It should be noted that the experimental $P b c a$ to $P 2_{1} / c$ phase transition occurs within $1.4 \mathrm{GPa}$ pressure window. The results of the PBE, LDA, and BLYP functionals predict that

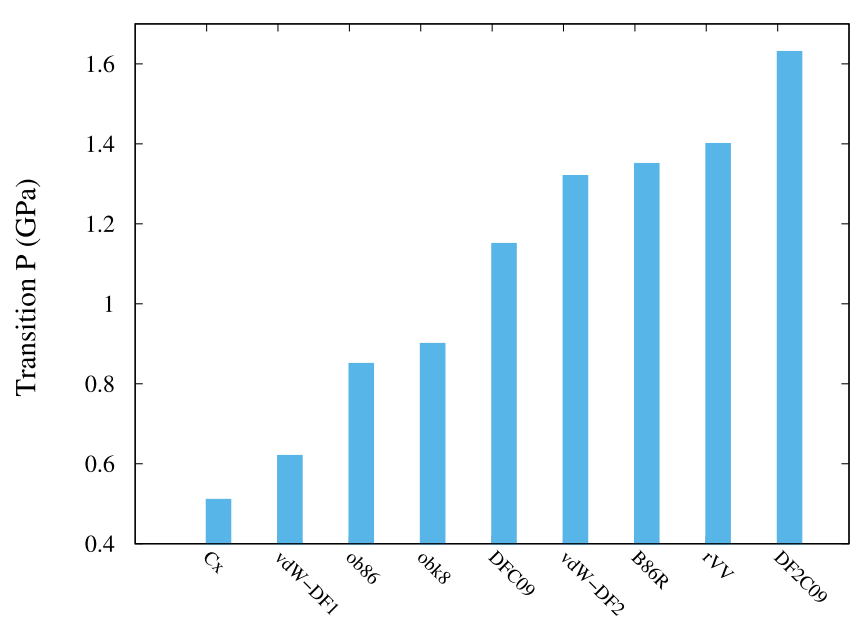

FIG. 6. $P b c a$ to $P 2_{1} / c$ phase transition pressure. The results are calculated using different vdW functionals as explained in text. the phase I-II transition occurs at 5.2, 5.6, and $3.5 \mathrm{GPa}$, respectively.

Our phase diagram calculations indicate that vdW results are in better agreement with experiments than the conventional functionals. Between the PBE and BLYP functionals, the PBE $\delta_{\mathrm{C}-\mathrm{C}}$ results are closer to vdW-DF1 and vdW-DF2 $\delta_{\mathrm{C}-\mathrm{C}}$ at low pressures. The difference between PBE phase transition pressure and vdW-DF1 and vdW-DF2 phase transition pressures is 4.55 and $3.9 \mathrm{GPa}$, respectively. However the difference between BLYP phase transition pressure and vdWDF1 and vdW-DF2 phase transition pressures is 2.85 and $2.2 \mathrm{GPa}$, respectively. As we discussed in Sec. III A, vdWDF1 and vdW-DF2 $\delta_{\mathrm{C}-\mathrm{C}}$ are positive for both $\mathrm{Pbca}$ and $P 2_{1} / C$ structures below $2 \mathrm{GPa}$ where the phase transition between them happens. Therefore the phase I-II transition in crystalline benzene occurs without any intermolecular contacts. This transition occurs only due to dispersion effects.

Using our DFT results, we compute the EOS of Pbca and $P 2_{1} / c$ structures. Figure 7 represents the results which are obtained by vdW and conventional functionals. We compare our DFT results with experiments which are reported in Refs. 9 and 11. The experimental results in Ref. 9 are $V(P)$ data for crystalline benzene at $540 \mathrm{~K}$ that have been fitted by the Vinet EOS. The second experimental results ${ }^{11}$ belong to crystalline benzene at lower pressures and $295 \mathrm{~K}$. Among DFT conventional functionals used in this study, only the PBE $V(P)$ results are close to experiments. The BLYP and LDA curves lie far above and below experimental curves, respectively. In general, the vdW results are in good agreement with experiments. At lower pressures vdW-DF1, ${ }^{52} \mathrm{vdW}-\mathrm{DF} 2,{ }^{50}$ and $\mathrm{DFcx}^{55} V(P)$ points for $P b c a$ phase are close to experiments. With increasing the pressure, the $P 2_{1} / c V(P)$ curves computed with vdW-DF2, ${ }^{50}$ obk $8,{ }^{35,53} \mathrm{rVV},{ }^{56,57}$ and $\mathrm{B} 86 \mathrm{R}^{35,53}$ are close to experimental points. The rVV functional has a different nonlocal correlation kernel, whereas other vdW functionals are the modified versions of vdW-DF1 or vdW-DF2. Our EOS calculations indicate that the modifications bring the vdW-DF1 and vdW-DF2 $V(P)$ curves below experimental ones. It is hard to conclude whether these modifications improve the accuracy of vdW-DF1 and vdW-DF2 functionals, especially in the case of vdW-DF2 functional, which overall gives the most accurate results. Our ground state EOS calculations indicate that at fixed pressure the volume per benzene molecule for $\mathrm{Pbca}$ 

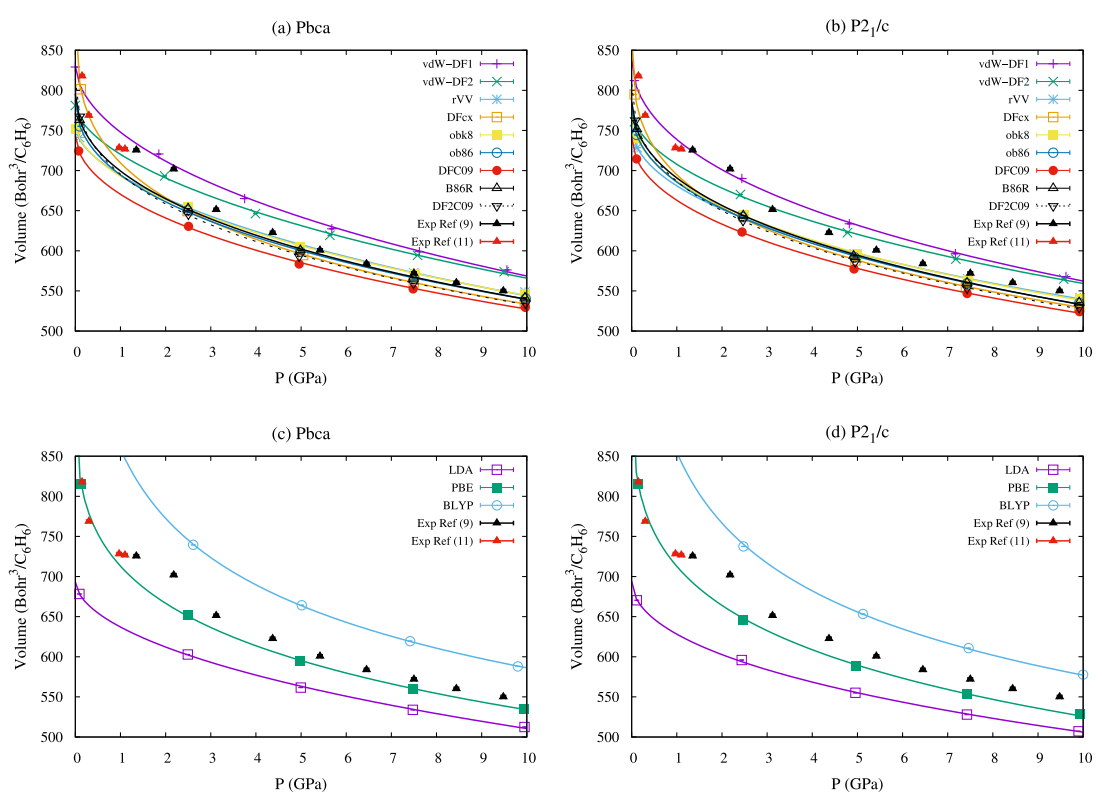

FIG. 7. EOS of $P b c a$ and $P 2_{1} / c$ structures obtained by vdW ((a) and (b)) and conventional ((c) and (d)) DFT functionals. The results are compared with experimental data which are reported in Refs. 9 and 11. phase is larger than $P 2_{1} / c$. This is in agreement with finite temperature experimental measurements. This conclusion is also independent of used DFT functionals.

To investigate the ZPE contribution in phase diagram calculations, we simulated the difference between the gas and crystal ZPEs. The ZPE of the $P b c a$ and $P 2_{1} / c$ structures with respect to gas phase is shown as function of pressure (Figure 8). We used the vdW-DF2 and vdW-DF1 functionals to optimise the structures for phonon calculations. ZPE is obtained using quasi-harmonic approximation, as explained in Sec. II. Within the studied pressure range, the difference between the ZPE of phases I and II is less than $2 \mathrm{meV} / \mathrm{atom}$. The vdW-DF2 results indicate that the phase I-II ZPE transition happens at $0.6 \mathrm{GPa}$, whereas the vdW-DF1 results predict that the phase I-II ZPE transition occurs at $1.65 \mathrm{GPa}$ (Figure 8). The difference between the ZPE of phases I and II increases with pressure. The ZPE correction to the cohesive energy of crystalline benzene was previously calculated. ${ }^{60}$ They evaluated the ZPE using $\Gamma$-point harmonic frequencies at the PBE level. They found that the ZPE of the Pbca structure is $44 \mathrm{meV} / \mathrm{molecule.} \mathrm{In} \mathrm{their} \mathrm{calculations,}$ they employed experimentally reported ${ }^{61}$ orthorhombic cell without full three-dimensional optimisation. Finitetemperature experiments ${ }^{62}$ show that the ZPE of crystalline benzene is $2.8 \mathrm{~kJ} / \mathrm{mol}$ ( $29.02 \mathrm{meV} /$ molecule). The $\mathrm{ZPE}$ experimental result is also employed to investigate the binding energy of benzene crystal. ${ }^{63}$ An estimate of $4.8 \mathrm{~kJ} / \mathrm{mol}$ was obtained using DFT many-body dispersion method. ${ }^{64}$ This ZPE is significantly larger than an estimate of $2.8 \mathrm{~kJ} / \mathrm{mol}$ which is obtained by finite molecular cluster calculations. ${ }^{12,65}$ Our ZPE results are close to PBC-DFT calculations, ${ }^{66}$ where an estimate of $2.6 \mathrm{~kJ} / \mathrm{mol}$ is obtained using the PBE functional.

The static phase diagrams in Figure 5 assume that the atoms are infinitely massive. We calculate the dynamic phase
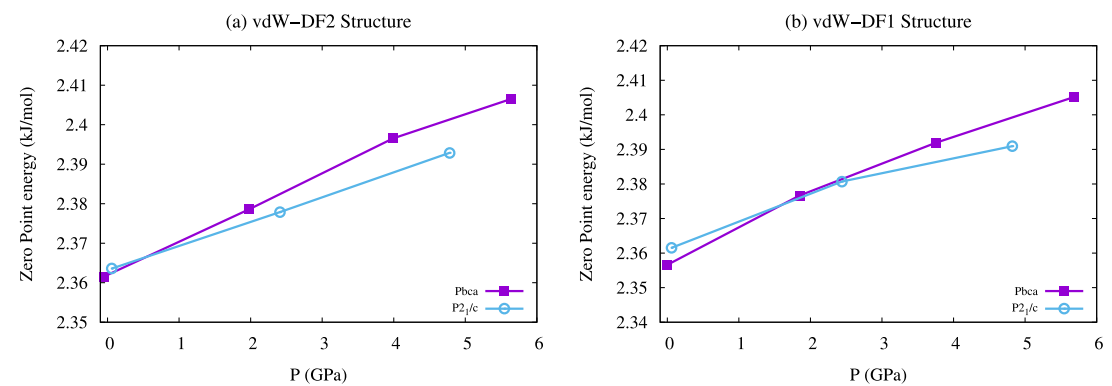

FIG. 8. ZPE of $P b c a$ and $P 2_{1} / c$ structures obtained by DFT. Geometries are accurately optimised by two functionals: (a) vdW-DF2 $2^{50}$ and (b) vdW-DF1. ${ }^{52}$
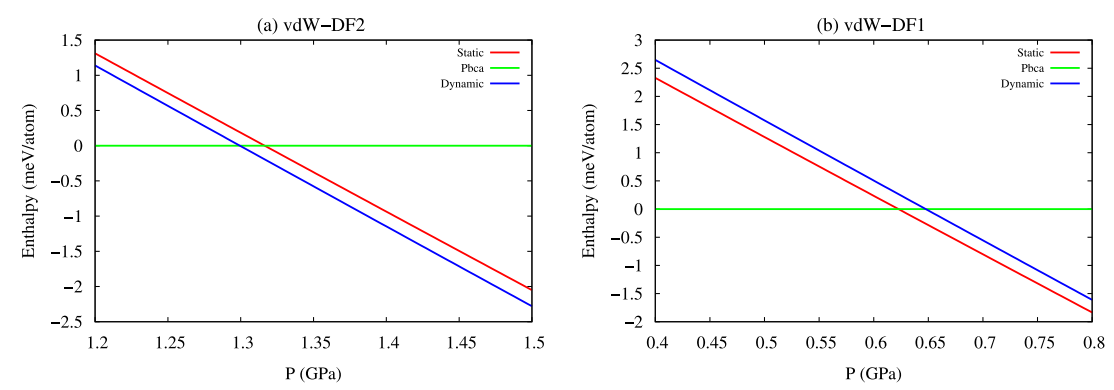

FIG. 9. Static and dynamic phase transition of $P b c a$ to $P 2_{1} / c$ obtained by (a) $\mathrm{vdW}-\mathrm{DF} 2^{50}$ and (b) vdW-DF1 ${ }^{52} \mathrm{vdW}$ functionals. 
TABLE I. DMC energies of the Pbca (phase I) structure. Energies are obtained at two simulation cells containing $N_{1}=48$ and $N_{2}=384$ atoms. Linear extrapolated energies are shown as $\mathrm{E}(\infty)$. Energy (E) and volume (Vol) are in $\mathrm{eV}$ and $\mathrm{Bohr}^{3}$ per benzene molecule, respectively.

\begin{tabular}{lccc}
\hline \hline Vol & $\mathrm{E}\left(N_{1}\right)$ & $\mathrm{E}\left(N_{2}\right)$ & $\mathrm{E}(\infty)$ \\
\hline 781.086 & $-1024.7464(4)$ & $-1022.7063(8)$ & $-1022.4140(8)$ \\
693.335 & $-1024.6376(5)$ & $-1022.5976(7)$ & $-1022.3052(7)$ \\
646.304 & $-1024.4880(5)$ & $-1022.4478(5)$ & $-1022.1556(5)$ \\
619.413 & $-1024.3588(4)$ & $-1022.3185(5)$ & $-1022.0264(5)$ \\
\hline \hline
\end{tabular}

diagram by adding the ZPE to the static results. Figure 9 illustrates the dynamic phase diagrams of crystalline benzene at the DFT level. The vdW-DF2 results indicate that adding ZPE lowers the phase transition by $0.02 \mathrm{GPa}$, and the $P b c a$ to $P 2_{1} / c$ phase transition pressure is $1.42 \mathrm{GPa}$. The vdW-DF1 results predict that the phase transition occurs at $0.66 \mathrm{GPa}$, which is $0.03 \mathrm{GPa}$ higher than the static phase transition pressure. The results of comparing the static and dynamic phase diagrams indicate that the ZPE contribution is negligible.

\section{Finite temperature DMC phase diagram}

In this section, we present our finite temperature phase diagram calculations. We use QMC based methods to calculate the electronic structure ground state energy. The inadequacy of mean-field-like DFT calculations of hydrogenrich systems was demonstrated before. ${ }^{67,68}$ To obtain reliable results, going beyond DFT-based methods and properly considering many-body effects are necessary. The DMC is generally considered as the most accurate first-principle method available in studying the phase diagram of hydrogendominant materials. ${ }^{31,32}$ In addition, DMC is an effective method to study non-covalent systems. It can reach and go beyond the chemical accuracy which is desired for noncovalent systems. ${ }^{16}$

We perform DMC calculations to obtain the wavefunction-based phase diagram for crystalline benzene at low-pressures. We use the vdW-DF2 optimized structure for our DMC calculations. As we demonstrated in our DFT
TABLE II. DMC energies of the $P 2_{1} / c$ (phase II) structure. Energies are obtained at two simulation cells containing $N_{1}=24$ and $N_{2}=192$ atoms. Linear extrapolated energies are shown as E( $\infty)$. Energy $(\mathrm{E})$ and volume (Vol) are in $\mathrm{eV}$ and $\mathrm{Bohr}^{3}$ per benzene molecule, respectively.

\begin{tabular}{lccc}
\hline \hline Vol & $\mathrm{E}\left(N_{1}\right)$ & $\mathrm{E}\left(N_{2}\right)$ & $\mathrm{E}(\infty)$ \\
\hline 760.8398 & $-1024.8824(5)$ & $-1022.6683(8)$ & $-1022.3514(8)$ \\
670.2382 & $-1024.7736(6)$ & $-1022.5582(8)$ & $-1022.2426(8)$ \\
622.5712 & $-1024.6240(5)$ & $-1022.4099(7)$ & $-1022.0929(8)$ \\
589.6330 & $-1024.4948(5)$ & $-1022.2793(8)$ & $-1021.9637(8)$ \\
\hline \hline
\end{tabular}

calculations, vdW-DF2 functional gives the closest results to experiment. The DMC results for energies in the limit of infinite system size are obtained by extrapolation using DMC energy data at $1 \times 1 \times 1$ and $2 \times 2 \times 2$ simulation cells.

Table I lists the DMC energies of the Pbca structure at four primitive unit-cell volumes. We consider two simulation cells for each density containing 48 and 384 atoms. DMC energy at thermodynamic limit is obtained by linear extrapolation in $1 / N$.

Table II shows the DMC energies of the $P 2_{1} / c$ structure at different primitive unit-cell volumes. We consider two simulation cells for each density containing 24 and 192 atoms. DMC energy at infinite system size limit is calculated by linear extrapolation in $1 / N$.

To identify enthalpy-pressure curves for the $P b c a$ and $P 2_{1} / c$ structures, we fitted model equations of state $E(V)$ to our finite-size-corrected DMC energy against volume $V$. We used the Vinet $\operatorname{EOS}^{51}$ to fit our total energies and propagate errors using classical statistics. The pressure $P=-(\partial E / \partial V)$ and the enthalpy is $H=E+P V$, where $E$ is DMC electronic structure energy of system.

Figure 10(a) illustrates the DMC energy of phases I and II of crystalline benzene as function of volume per benzene molecule. The inset illustrates the DMC pressure as function of volume for $P b c a$ and $P 2_{1} / c$ structures. With increasing density, phase II becomes favourable over phase I in the Pbca structure. Figure 10(b) shows the relative enthalpies of the $P b c a$ and $P 2_{1} / c$ structures. Based on our static enthalpypressure phase diagram, the $P b c a$ to $P 2_{1} / c$ phase transition occurs at pressure $2.6 \pm 0.1 \mathrm{GPa}$. The use of the DMC

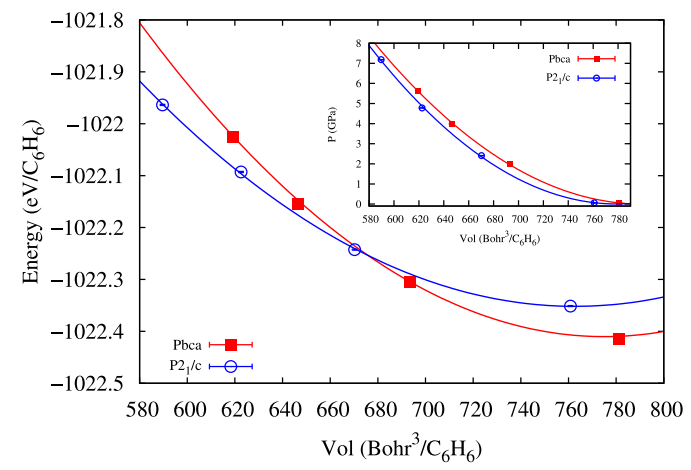

(a)

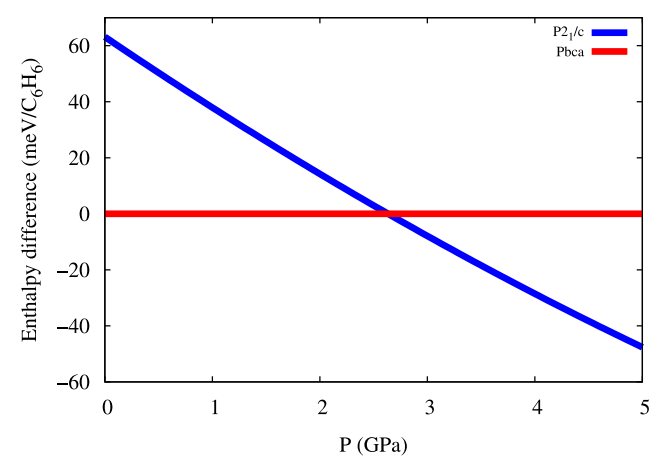

(b)

FIG. 10. (a) DMC energy of the Pbca and $P 2_{1} / c$ structures as function of volume per benzene molecule. The inset shows the DMC pressure as function of volume. Energy error bars are included in point sizes and are of the order of meV. (b) Relative enthalpies of the $P b c a$ and $P 2_{1} / c$ structures as function of pressure. The widths of the DMC lines indicate the estimated uncertainties in the enthalpies because of statistical and systematic errors. 

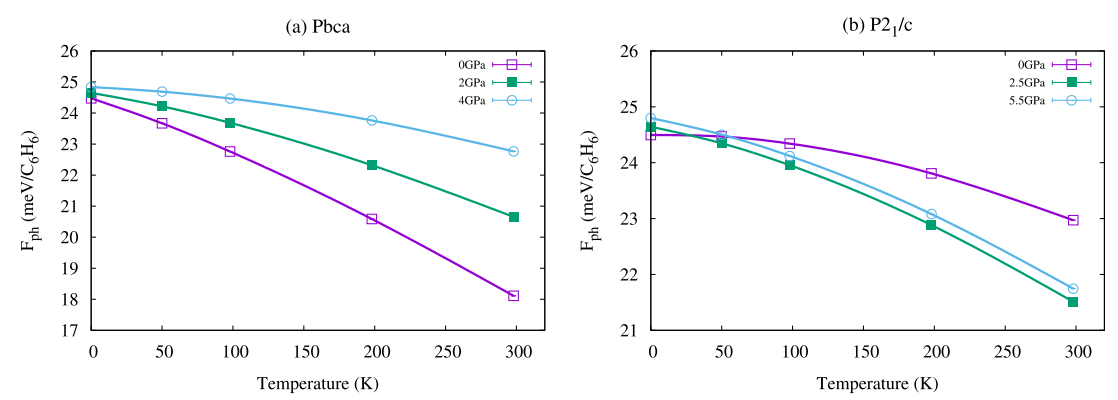

FIG. 11. Phonon contribution to the Helmholtz free energies $F_{p h}$ of the $P b c a$ and $P 2_{1} / c$ structures of crystalline benzene. The geometries are optimized using vdW-DF2 $2^{50}$ functionals.
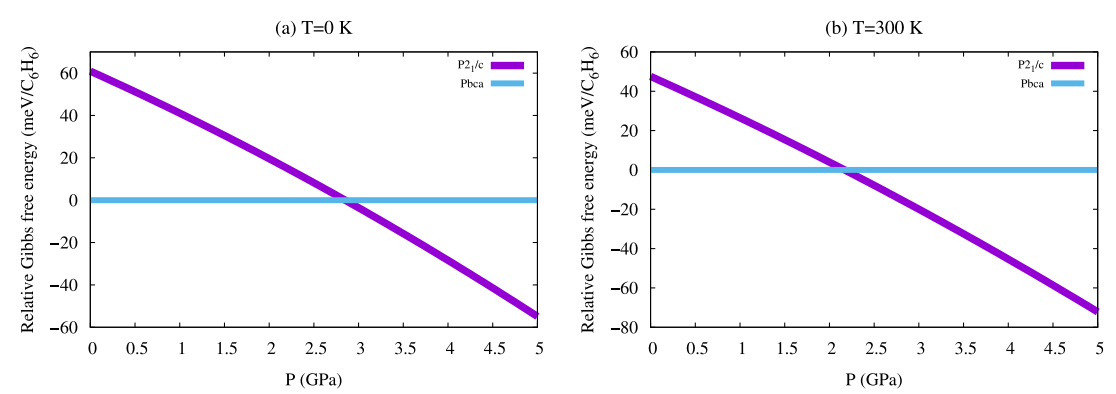

FIG. 12. Relative Gibbs free energies of the $P b c a$ and $P 2_{1} / c a$ structures. (a) $0 \mathrm{~K}$ and (b) $300 \mathrm{~K}$. The Gibbs free energies are calculated using staticlattice DMC calculations together with DFT quasi-harmonic vibrational calculations.

method has significant consequences for the static-lattice relative enthalpies of the studied structures. Compared with vdW-DF2, the DMC enthalpy-pressure results predict that the phase I-II transition occurs at $1.2 \mathrm{GPa}$ higher pressure. Among conventional DFT functionals, the BLYP results are closest to DMC. The difference between DMC and BLYP phase transition pressure is $0.9 \mathrm{GPa}$.

To obtain the phase diagram at finite temperature, we used quasi-harmonic approximation to obtain lattice dynamic contribution to the free energies. Phonons have contributed to the Helmholtz free energies $F_{p h}$ of crystalline benzene (Figure 11). We used vdW-DF2 functionals to optimise the $P b c a$ and $P 2_{1} / c$ structures at different pressures. Vibrational free energies are calculated at different temperatures of 50, 100,200 , and $300 \mathrm{~K}$. At room temperature and $0 \mathrm{GPa}$, vibrational free energy of $P 2_{1} / c$ is higher than $P b c a$. Meanwhile, the vibrational free energies of $P b c a$ become higher than $P 2_{1} / c$ by increasing the pressure. These results indicate the stability of the $\mathrm{Pbca}$ phase at ambient conditions, which is also observed experimentally. 9,10

We calculated relative Gibbs free energies of the Pbca and $P 2_{1} / c$ structures at room temperature (Figure 12). The static electronic structure results are obtained by DMC calculations. Our results predict that the room temperature $\mathrm{Pbca}$ to $P 2_{1} / \mathrm{c}$ structure transformation happens at 2.1(1) GPa. Experiments indicate that the room temperature phase transition of $\mathrm{Pbca}$ to $P 2_{1} / c$ occurs at around $1.4 \mathrm{GPa}^{3}$ The I to II phase transition is a temperature dependent transformation, and it can be speeded up by heating the sample. ${ }^{1}$ Keeping the low-pressure phase I, $\mathrm{Pbca}$, in a metastable state at least up to $3 \mathrm{GPa}$ is possible without heating. ${ }^{5}$ Our zero temperature DMC phase diagram predicts that the Pbca phase could be stable up to 2.6(1) GPa (Figure 12(a)). The phase diagram that we have obtained by combining DMC static-lattice energies and quasi-harmonic vibrational energies can be extended to higher pressures.

As the final step of our study, we calculated the lattice energy of crystalline benzene at ambient conditions. The cohesive energy yields the strength of the vdW forces holding the crystalline benzene together. We used our DMC and ZP energies for $P b c a$ structure. The cohesive energy is calculated using the difference between total energies of $\mathrm{Pbca}$ structure and its fragments. Cohesive energy calculation is a precise test of DMC method, since it has to accurately describe two different systems of benzene molecule and crystalline benzene. The electronic structure of these two systems are not similar. In our DMC lattice energy calculation, we used same time step of 0.01 a.u. for both crystal and molecule. We found an estimate of $50.6 \pm 0.5 \mathrm{~kJ} / \mathrm{mol}$ for lattice energy. Ab initio many-electron wave functions methods provide an estimate of $55.90 \pm 0.76 \mathrm{~kJ} / \mathrm{mol}$ for benzene crystal lattice energy at zero temperature. ${ }^{15}$ The experimental lattice energy at same condition is $55.3 \pm 2.2 \mathrm{~kJ} / \mathrm{mol} .{ }^{15} \mathrm{We}$ used conventional Jastrow factor in our DMC calculations. In principle, the DMC lattice energy can be systematically improved by accurately taking into account the correlation energy and also decreasing the fixed-node errors. These purposes can be fulfilled by adding additional terms in Jastrow factor and using backflow transformations. ${ }^{16}$ However, improving the DMC lattice energy until it converges to exact results requires huge amount of computational time.

\section{CONCLUSION}

We have comprehensively studied the crystalline benzene phase diagram at pressures below $10 \mathrm{GPa}$. We have used different vdW functionals and also three most used conventional functionals to obtain the DFT energy of the system. The vdW-DF2 results of our study indicated that the $P b c a$ and $P 2_{1} / c$ structures are the best candidates for phases I and II, respectively. We have used the accurate DMC method to calculate the ground-state electronic structure energy of system. We have compared static enthalpy-pressure phase diagrams which are obtained by DFT and DMC methods. We used quasi-harmonic approximation and density functional 
perturbation theory to calculate the phonon contribution to the free energy of the system. Our Gibbs free energy phase diagram predicts that at room temperature, the phase I-II transition occurs at 2.1(1) GPa, which is $0.7(1) \mathrm{GPa}$ higher than experimental result. ${ }^{3}$ Our DMC energies at the thermodynamic limit are calculated using $1 \times 1 \times 1$ and $2 \times 2 \times 2$ cell extrapolation. Improving the finite size extrapolation may reduce the difference between our DMC Gibbs free energy and experiment. The other source of this difference could be the quasi-harmonic approximation which has been used in this work. Anharmonic contribution plays an important role in the study of phase diagram of hydrogen-dominant materials and can affect the phase transition pressure. ${ }^{31,32}$ We have found DMC lattice energy of $50.6 \pm 0.5 \mathrm{~kJ} / \mathrm{mol}$ for crystalline benzene at ambient conditions. The results of our study indicate the importance of many-body electronic structure calculation to obtain a reliable phase diagram for molecular crystals.

\section{ACKNOWLEDGMENTS}

This study utilised computing facilities provided by ARCHER, the UK national super computing service, and by the University College London high-performance computing centre. S. Azadi acknowledges that the results of this research have been obtained using the PRACE-3IP project (No. FP7 RI-312763) resource ARCHER based in the UK. The authors acknowledge the financial support of the European Research Council under the Advanced Grant ToMCaT (Theory of Mantle, Core, and Technological Materials). R. E. Cohen acknowledges the support of the Carnegie Institution for Science.

${ }^{1}$ M. M. Thièry and J. M. Lèger, J. Chem. Phys. 89, 4255 (1988).

${ }^{2}$ X.-D. Wen, R. Hoffmann, and N. W. Ashcroft, J. Am. Chem. Soc. 133, 9023 (2011).

${ }^{3}$ G. J. Piermarini, A. D. Mighell, C. Weir, and S. Block, Science 165, 3461 (1969).

${ }^{4}$ P. Raiteri, R. Martonak, and M. Parrinello, Angew. Chem., Int. Ed. 44, 3769 (2005).

${ }^{5}$ P. W. Bridgman, J. Chem. Phys 9, 794 (1941).

${ }^{6}$ E. G. Cox and J. A. S. Smith, Nature 173, 75 (1954).

${ }^{7}$ E. G. Cox, D. W. Cruickshank, and J. A. S. Smith, Proc. R. Soc. A 247, 1 (1958).

${ }^{8}$ G. E. Bacon, N. A. Curry, and S. A. Wilson, Proc. R. Soc. A 279, 98 (1964).

${ }^{9}$ L. Ciabini, F. A. Gorelli, M. Santoro, R. Bini, V. Schettino, and M. Mezouar, Phys. Rev. B 72, 094108 (2005).

${ }^{10}$ L. Ciabini, M. Santoro, F. A. Gorelli, R. Bini, V. Schettino, and S. Raugei, Nat. Mater. 6, 39 (2007).

${ }^{11}$ A. Katrusiak, M. Podsiadzo, and A. Budzianowski, Cryst. Growth Des. 10, 3461 (2010).

${ }^{12}$ R. Podeszwa, B. M. Rice, and K. Szalewicz, Phys. Rev. Lett. 101, 115503 (2008).

${ }^{13}$ S. Grimme, J. Antony, S. Ehrlich, and H. Krieg, J. Chem. Phys. 132, 154104 (2010).

${ }^{14}$ J. P. Perdew, K. Burke, and M. Ernzerhof, Phys. Rev. Lett. 77, 3865 (1996).

${ }^{15}$ J. Yang, W. Hu, D. Usvyat, D. Matthews, M. Schutz, and G. K.-L. Chan, Science 345, 640 (2014).

${ }^{16}$ S. Azadi and R. E. Cohen, J. Chem. Phys. 143, 104301 (2015).

${ }^{17}$ W. M. C. Foulkes, L. Mitas, R. J. Needs, and G. Rajagopal, Rev. Mod. Phys. 73, 33 (2001)

${ }^{18}$ M. Marchi, S. Azadi, M. Casula, and S. Sorella, J. Chem. Phys. 131, 154116 (2009).
${ }^{19}$ M. D. Brown, J. R. Trail, P. L. Ríos, and R. J. Needs, J. Chem. Phys. 126, 224110 (2007).

${ }^{20}$ I. G. Gurtubay and R. J. Needs, J. Chem. Phys. 127, 124306 (2007).

${ }^{21}$ J. R. Trail and R. J. Needs, J. Chem. Phys. 128, 204103 (2008).

${ }^{22}$ S. Azadi, R. Singh, and T. D. Kuhne, Int. J. Quantum Chem. 115, 1673 (2015).

${ }^{23}$ M. Marchi, S. Azadi, and S. Sorella, Phys. Rev. Lett. 107, 086807 (2011).

${ }^{24}$ E. Mostaani, N. Drummond, and V. Falko, Phys. Rev. Lett. 115, 115501 (2015).

${ }^{25}$ S. Azadi and W. M. C. Foulkes, J. Chem. Phys. 143, 102807 (2015).

${ }^{26}$ S. J. Cox, M. D. Towler, D. Alfè, and A. Michaelides, J. Chem. Phys. 140, 174703 (2014).

${ }^{27}$ J. Ma, A. Michaelides, and D. Alfè, J. Chem. Phys. 134, 134701 (2011).

${ }^{28}$ Y. S. Al-Hamdani, D. Alfè, and O. A. von Lilienfeld, J. Chem. Phys. 141, 18C530 (2014).

${ }^{29}$ N. A. Benedek, I. K. Snook, M. D. Towler, and R. J. Needs, J. Chem. Phys. 125, 104302 (2006).

${ }^{30}$ M. Dubecký, L. Mitas, and P. Jurečka, Chem. Rev. 116, 5188 (2016).

${ }^{31}$ N. Drummond, B. Monserrat, J. Lloyd-Williams, P. L. Rìos, and R. J. Needs, Nat. Commun. 6, 7749 (2015).

${ }^{32}$ S. Azadi, B. Monserrat, W. M. C. Foulkes, and R. J. Needs, Phys. Rev. Lett. 112, 165501 (2014).

${ }^{33}$ S. Azadi, W. M. C. Foulkes, and T. D. Kuhne, New J. Phys. 15, 113005 (2013).

${ }^{34}$ E. Miliordos, E. Aprà, and S. S. Xantheas, J. Phys. Chem. A 118, 7568 (2014).

35J. Klimeš and A. Michaelides, J. Chem. Phys. 137, 120901 (2012).

${ }^{36}$ P. Giannozzi et al., J. Phys.: Condens. Matter 21, 395502 (2009).

${ }^{37}$ A. M. Rappe, K. M. Rabe, E. Kaxiras, and J. D. Joannopoulos, Phys. Rev. B 41, 1227 (1990).

${ }^{38}$ S. Baroni, S. de Gironcoli, and A. D. Corso, Rev. Mod. Phys. 73, 515 (2001).

${ }^{39}$ R. J. Needs, M. D. Towler, N. D. Drummond, and P. L. Ríos, J. Phys.: Condens. Matter 22, 023201 (2010).

${ }^{40}$ J. R. Trail and R. J. Needs, J. Chem. Phys. 122, 174109 (2005).

${ }^{41}$ J. R. Trail and R. J. Needs, J. Chem. Phys. 122, 014112 (2005).

${ }^{42}$ L. Mitas, E. L. Shirley, and D. M. Ceperley, J. Chem. Phys. 95, 3467 (1991).

${ }^{43}$ C. W. Greeff and W. A. Lester, Jr., J. Chem. Phys. 109, 1607 (1998).

${ }^{44}$ J. M. Zuo, P. Blaha, and K. Schwarz, J. Phys.: Condens. Matter 9, 7541 (1997).

${ }^{45}$ S. Azadi, C. Cavazzoni, and S. Sorella, Phys. Rev. B 82, 125112 (2010).

${ }^{46}$ D. Alfè and M. J. Gillan, Phys. Rev. B 70, 161101 (2004).

${ }^{47}$ C. J. Umrigar, K. G. Wilson, and J. W. Wilkins, Phys. Rev. Lett. 60, 1719 (1988).

${ }^{48}$ N. D. Drummond and R. J. Needs, Phys. Rev. B 72, 085124 (2005).

${ }^{49}$ J. Perdew, A. Ruzsinszky, G. I. Csonka, O. A. Vydrov, G. E. Scuseria, L. A. Constantin, X. Zhou, and K. Burke, Phys. Rev. Lett. 100, 136406 (2008).

${ }^{50}$ K. Lee, E. D. Murray, L. Kong, B. I. Lundqvist, and D. C. Langreth, Phys. Rev. B 82, 081101(R) (2010).

${ }^{51}$ P. Vinet, J. Ferrante, J. R. Smith, and J. H. Rose, J. Phys. C: Solid State 19, L467 (1986)

${ }^{52}$ M. Dion, H. Rydberg, E. Schröder, D. C. Langreth, and B. I. Lundqvist, Phys. Rev. Lett. 92, 246401 (2004).

${ }^{53}$ J. Klimeš, D. R. Bowler, and A. Michaelides, Phys. Rev. B 83, 195131 (2012).

${ }^{54}$ V. R. Cooper, Phys. Rev. B 81, 161104(R) (2010).

${ }^{55}$ K. Berland and P. Hyldgaard, Phys. Rev. B 89, 035412 (2014).

${ }^{56}$ R. Sabatini, T. Gorni, and S. de Gironcoli, Phys. Rev. B 87, 041108(R) (2013).

${ }^{57}$ O. A. Vydrova and T. V. Voorhis, J. Chem. Phys. 133, 244103 (2010).

${ }^{58}$ J. P. Perdew and A. Zunger, Phys. Rev. B 23, 5048 (1981).

${ }^{59}$ C. Lee, W. Yang, and R. G. Parr, Phys. Rev. B 37, 785 (1988).

${ }^{60}$ O. Bludský and M. Rubeš, Phys. Rev. B 77, 092103 (2008).

${ }^{61}$ W. I. F. David, R. M. Ibberson, G. A. Jeffrey, and J. R. Ruble, Physica B 180, 597 (1992).

${ }^{62}$ M. Nakamura and T. Miyazawa, J. Chem. Phys. 51, 3146 (1969).

${ }^{63}$ D. Lu, Y. Li, D. Rocca, and G. Galli, Phys. Rev. Lett. 102, 206411 (2009).

${ }^{64}$ A. M. Reilly and A. Tkatchenko, J. Chem. Phys. 139, 024705 (2013).

${ }^{65}$ A. L. Ringer and C. D. Sherrill, Chemistry 14, 2542 (2008).

${ }^{66}$ A. O. de-la Roza and E. R. Johnson, J. Chem. Phys. 137, 054103 (2012).

${ }^{67}$ S. Azadi and W. M. C. Foulkes, Phys. Rev. B 88, 014115 (2013).

${ }^{68}$ N. D. Drummond, R. J. Needs, A. Sorouri, and W. M. C. Foulkes, Phys. Rev. B 78, 125106 (2008). 